\title{
An Improved Iterative Segmentation Algorithm using Canny Edge Detector with Iterative Median Filter for Skin Lesion Border Detection
}

\author{
J. H. Jaseema Yasmin \\ Research Scholar, Department of Computer \\ Science and Engineering, Periyar Maniammai \\ University, Vallam, Thanjavur, India
}

\author{
M. Mohamed Sadiq \\ Principal, Sadakathullah Appa College, Tirunelveli, \\ India
}

\begin{abstract}
A system for the computer-aided diagnosis of melanoma, provides quantitative and objective evaluation of the skin lesion, as opposed to visual assessment, which is subjective in nature, is comprised of four major components: skin image acquisition, lesion segmentation, feature extraction, and lesion classification. Automatic segmentation of lesions in color skin images, which is the main focus of this paper, is one of the most important steps towards the automated analysis and evaluation of dermoscopy images in the computer aided diagnosis of melanoma. The accuracy of segmentation is highly dependent on the success or failure of each computerized analysis procedure. An improved iterative segmentation algorithm using canny edge detector with iterative median filter, for border detection of real skin lesions is presented,which helps in early detection of malignant melanoma and its performance is compared with the segmentation algorithm using canny detector [1] developed by us previously for border detection of real skin lesions. The experimental results demonstrate the successful border detection of noisy real skin lesions by the proposed segmentation algorithm. We conclude that the proposed, improved iterative segmentation algorithm using canny detector with iterative filtering, segments the lesion from the image even in the presence of noise for a variety of lesions, and skin types and its performance is better than the segmentation algorithm [1] that we have developed previously that uses canny detector, for border detection of real skin lesions.
\end{abstract}

\section{General Terms}

Pattern Recognition, Digital Image Processing, Algorithms.

\section{Keywords}

Image Segmentation, Skin Lesion, Canny detector, Border detection, Iterative filter, Melanoma.

\section{INTRODUCTION}

A set of regions that collectively cover the entire image, or a set of contours extracted from the image is the result of image segmentation. With respect to some characteristic or computed property, such as color, intensity, or texture, all of the pixels in a region are similar. With respect to the same characteristics adjacent regions are significantly different [2]. An object may never be recognizable, without a good segmentation algorithm. Under segmenting an image will group various objects into one region, while over segmenting it will split an object into different regions. In this way, the eventual success or failure of the analysis is determined by segmentation. For this reason, considerable care is taken, to improve the state of the art in color image segmentation [3].
At present, data visualization is one of the hotspots of image processing research, especially visualization of medical image (such as X-ray, Computer Tomography (CT), Magnetic Resonance Image (MRI) and Position Emission Tomography (PET)) [4]. Edge detection is one of the most frequently used techniques in digital image processing. The boundaries of object surfaces in a scene often show the way to oriented localized changes in intensity of an image, called edges. There is a long search for a good edge detection algorithm to use in image processing due to this observation combined with a commonly held belief that edge detection is the first step in image segmentation [5].For color image segmentation, several approaches of different complexity already exist. There is no general technique that can solve all the different image segmentation types, until now. Generally, the four categories of image segmentation approaches are thresholding, clustering, edge detection, and region extraction [6].

Over the past decades, malignant melanoma is the deadliest type of skin cancer and its incidence and mortality rates have been steadily increasing worldwide. Computer aided diagnosis of melanoma, as opposed to visual assessment, which is subjective in nature, provides quantitative and objective evaluation of the skin lesion. By diminishing the inter-observer and intra-observer variabilities that could be found in dermatologists examinations, it allows for reproducible diagnosis. It also automates the analysis, and thereby reduces the amount of repetitive and tedious tasks to be done by physicians [7]. Since melanoma can be cured with a simple excision if detected early, early diagnosis is particularly important.

The detection of the lesion borders is the first step in the computerized analysis of skin lesion images. The border structure offers important information for accurate diagnosis. From the border, many clinical features such as asymmetry, border irregularity etc are calculated [8]. Stages of the process of melanoma skin cancer diagnosis are preprocessing, segmentation, $\mathrm{ABCD}$ feature extraction from the lesion, and the calculation of Total Dermatoscopic Value (TDV) [9]. The rest of the paper is organized as follows: section 2 states about the related work regarding this topic, section 3 states the proposed methodology, section 4 demonstrates the results to show the effectiveness of new method and the conclusion is drawn in section 5 .

\section{PREVIEW OF RELATED WORK}

In medical image analysis image segmentation is a vital task. By minimizing the effect of noise, intensity inhomogeneity, and other factors in low-level image signals, the main challenge is to retrieve high-level information from low-level image signals. However, because of the diversity and 
complexity of images, the design of robust and efficient segmentation algorithm is still a very challenging research topic [17]. A variety of image segmentation methods have been proposed for this purpose, to address the challenges.

Meng-Husiun Tsai et al. developed a cytoplast and nucleus contour (CNC) detector to split the nucleus and cytoplast from a cervical smear image. This paper proposes the bi-group enhancer to make a clear-cut separation for the pixels laid between two objects, and the maximal color difference (MCD) method to draw the aptest nucleus contour. The CNC detector adopts a median filter to eradicate noises, the bigroup enhancer to restrain the noises and brighten the object contours, the K-mean algorithm to differentiate the cytoplast from the background, and the MCD method to extract the nucleus contour [10].

F. Ercal, proposed, a simple and yet effective method to find the borders of tumors as an initial step towards the diagnosis of skin tumors from their color images. The method makes use of an adaptive color metric from the red, green, and blue (RGB) planes that contain information to discriminate the tumor from the background. Using this suitable coordinate transformation, the image is segmented. The tumor portion is then extracted from the segmented image and borders are drawn [11]

L. Xu et al. developed a three-step segmentation method using the properties of skin cancer images. The steps of their method are as follows: 1. Pre-processing: a color image is first converted into an intensity image in such a way that the intensity at a pixel illustrations the color distance of that pixel with the color of the background. The median color of pixels in small windows in the four corners of the image is taken to be the color of the background. 2. Initial segmentation: a threshold value is determined from the average intensity of high gradient pixels in the obtained intensity image. To find approximate lesion boundaries, this threshold value is used. 3 . Region refinement: Using edge information in the image, a region boundary is refined. This involves initializing a closed elastic curve at the approximate boundary, and shrinking and expanding it to fit to the edges in its neighbourhood [12].

Rahil Garnavi et al. proposed a novel automatic segmentation algorithm using color space analysis and clustering-based histogram thresholding, which is able to find out the optimal color channel for segmentation of skin lesions [7].

With copious methods reported, image segmentation is conceivably, the most premeditated area in computer vision. The algorithm will have to be able to confiscate noise and other undesired features in the image, and to correctly segment the lesion. Over the last decade, developing robust and proficient algorithm for medical image segmentation has been an exigent area of interesting research interest [13].

An efficient and robust segmentation algorithm against noise is needed for medical image segmentation. Accurate segmentation of medical images is therefore highly challenging, however, accurate segmentation of these images is imperative in correct diagnosis by clinical tools [14].

Maciel Zortea et al. proposed a segmentation methodology which is characterized by three main processing stages:

1) Initialization: Under assumptions on the approximate location of the lesion, and the usual lighter nature of the skin color compared to the lesion, automatic quest for seed regions are done. The seed regions provide training samples for the binary classification between background skin and lesion.

2) Classification: A linear and quadratic classifier, the two distinct base classifiers are made available to the classification procedure. Using optimization of the classification accuracy, the decision of which classifier to use for each particular lesion, or possibly a combination of both classifiers (weighting), is automatically decided. This classification strategy can be seen as a hybrid classifier, here defined in terms of the combination of the posterior classification probabilities. To facilitating robust selection of new training samples and segmentation, the classification is iterated.

3) Iteration: The automatically chosen training samples are updated and the classification is repeated iteratively until convergence [15].

We have previously developed a segmentation algorithm [1], to extract the true border that reveals the global structure irregularity, which may evoke excessive cell growth or regression of a melanoma. The steps of this algorithm are as follows: 1.This algorithm is applied to the input image containing the lesion, where the input RGB image is converted to grayscale image. 2. Salt and pepper noise is added to the grayscale image and background noise reduction techniques are used to filter noise. 3. The noise filtered image is converted to a binary image, based on threshold. 4 . Then the binary image is converted to xor image. 5. The canny Edge detector is used to find the edges in the xor image .We get the edge detected image. 6. The pixel on the border of the object is found. 7. Using this pixel found on the border of the object (Lesion) as the starting pixel, the border of the lesion is traced, using the segmentation algorithm using canny detector [1].

In this paper, we have developed and compared the performance of our proposed methodology, the improved iterative segmentation algorithm using canny detector with iterative filtering for border detection of real skin lesions for noisy skin lesion images, with the segmentation algorithm using canny detector for border detection of real skin lesions for noisy skin lesion images developed by us previously [1].

\section{PROPOSED METHODOLOGY}

In order to separate the lesion from the surrounding normal skin, a new improved iterative segmentation algorithm, to detect the border of the lesion, has been developed and discussed in this paper. This algorithm is applied to the image containing the lesion. The proposed algorithm consists of several steps, which are explained below.

\subsection{An Improved Iterative Segmentation Algorithm using Canny Edge Detector with Iterative Median Filtering for Skin Lesion Border Detection}

Step 1: The RGB image is converted to grayscale image

Step 2: Salt and pepper noise is added to the grayscale image .The noisy image is the input image.

Step 3: Iterative Median filter used as the background noise reduction technique to filter noise.

Step 4: After noise reduction, the image is converted to a black and white image, based on threshold

Step 5: The black and white image got is converted into xor image

Step 6: The canny edge detector is used to find the edges in the xor image. We get the edge detected image (Fig 1(h))

Step 7: Creation of skin lesion Border Traced image in any 
of the iterations from 1 to $n$.

After getting the edge detected image, then for each iteration $\mathrm{i}$, for $\mathrm{i}=1$ to $\mathrm{n}$,

$\mathrm{n}=2,3,4, \ldots \ldots$. the border of the object (Lesion) is traced. The border traced image is got as output for iterations $\mathrm{i}=1$ to $\mathrm{n}$, as following:

\section{Step7.1: For iteration 1}

In the first iteration the edge detected image shown in Fig 1(h), is given as input to the canny edge detector. We get the edge detected image for the iteration 1 as shown in Fig 1(i). Next the pixel on the border of the object is found. For that the row and column co-ordinate of the pixel on the border of the lesion is to be found. To find the row co-ordinate of the pixel on the border of the object the black and white image is used. And the row co-ordinate of the pixel on the border of the object is found. And to find the the column co- ordinate of the pixel on the border of the object to be traced, the edge detected image got for iteration 1(Fig 1(i)) is used. To find the column co-ordinate, for the selected row, indices and values of non zero elements are found and the indices having the minimum value is selected as the column co- ordinate of the pixel on the border. Now the row and column co-ordinates of the pixel on the border of the object is found. Using this pixel found on the border of the object (Lesion) as the starting pixel, the border of the lesion is traced for iteration 1, using the improved iterative segmentation algorithm using canny detector, as shown in Fig 1(j), successfully.

\section{Step 7.2: For iteration 2 to $n$}

For iterations 2 to $n$, the output of the canny edge detector in the previous iterations (Iteration 1 to Iteration (n1)) are given as input to the canny edge Detector. Next the pixel on the border of the object is found as said above. Using this pixel found on the border of the object (Lesion) as the starting pixel, the border of the lesion is traced for each iteration ' $\mathrm{i}$ ', for $\mathrm{i}=2$ to $\mathrm{n}$, using the improved iterative segmentation algorithm using canny detector with iterative filtering, successfully and the processs ends when

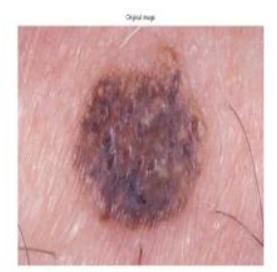

(a)

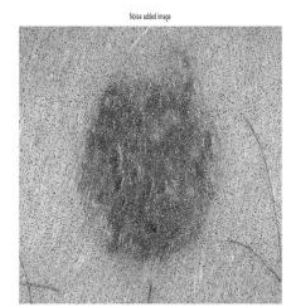

(c)

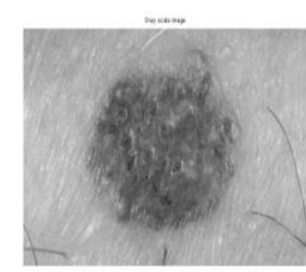

(b)

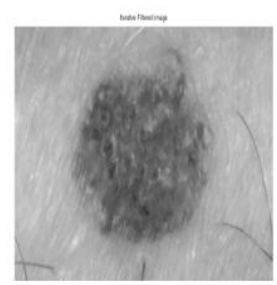

(d)

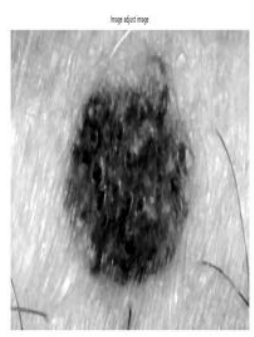

(e)

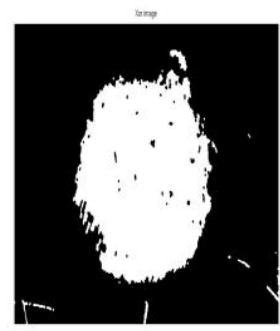

(g)

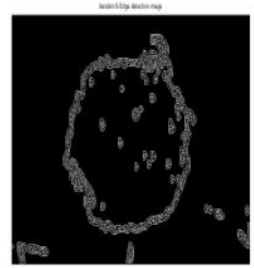

(i)

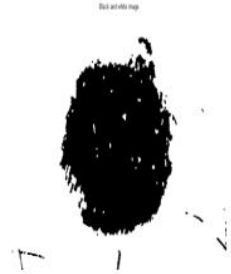

(f)

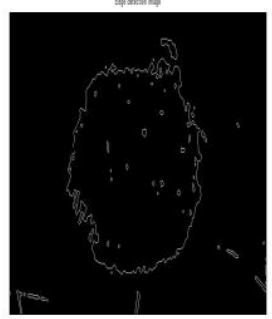

(h)

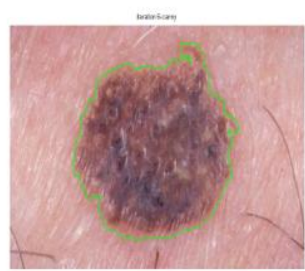

(j)
Fig 1: Border detection sequence using the proposed Improved Iterative Segmentation Algorithm Using Canny Edge Detector with iterative filtering

(a)Original Image (b) Gray Scale Image (c) Noise added Image (d) Iterative Filtered Image (e) Intensity Adjusted Image (f) Black and White Image (g) Xor image (h) Edge Detected Image (i) Edge Detected Image of Iteration 5 (j) Border detected Image using the Proposed Segmentation Algorithm

$\mathrm{i}>\mathrm{n}$. The Border detection sequence using the proposed Improved Iterative Segmentation Algorithm Using Canny Edge Detector with iterative filtering is shown in Fig 1.

\section{RESULTS AND DISCUSSION}

An image segmentation algorithm to extract the border of the skin lesions has been implemented using Matlab.We tested our border finding technique on original skin lesion images shown in Fig 2

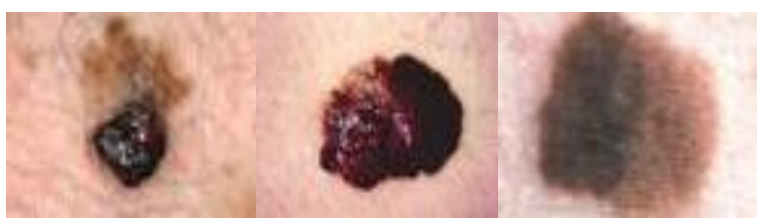



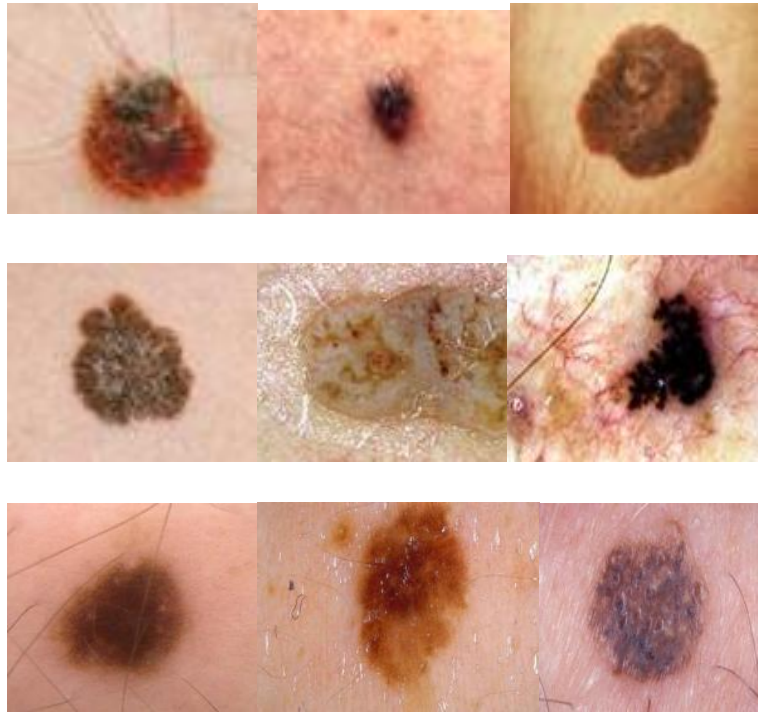

Fig 2: Original images referred from $[8],[9],[16],[17],[18]$
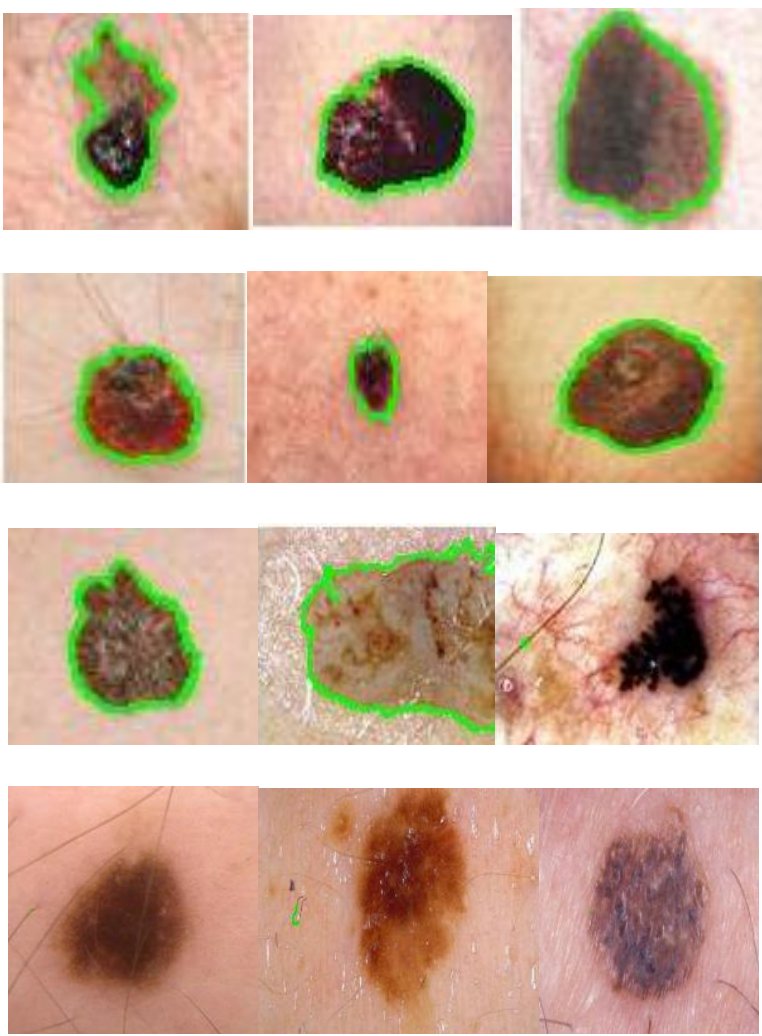

(a)

Fig 3a. Border detection results of various original skin lesions shown in Figure 2

(a) By the Segmentation Algorithm Using canny Edge Detector [1] for various original skin lesions

iteration 1-canny

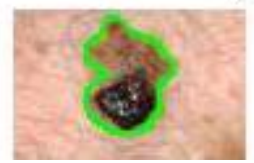

iteration 1-canny

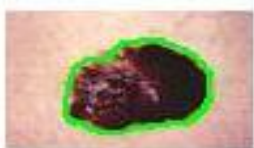

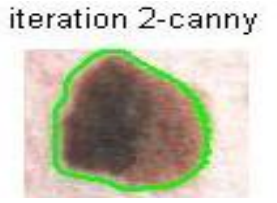

iteration 1-canny

iteration 1-canny

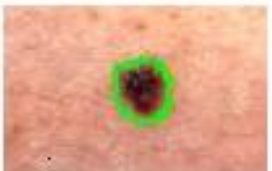

iteration 1-canny

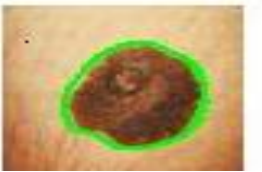

iteration 1-canny
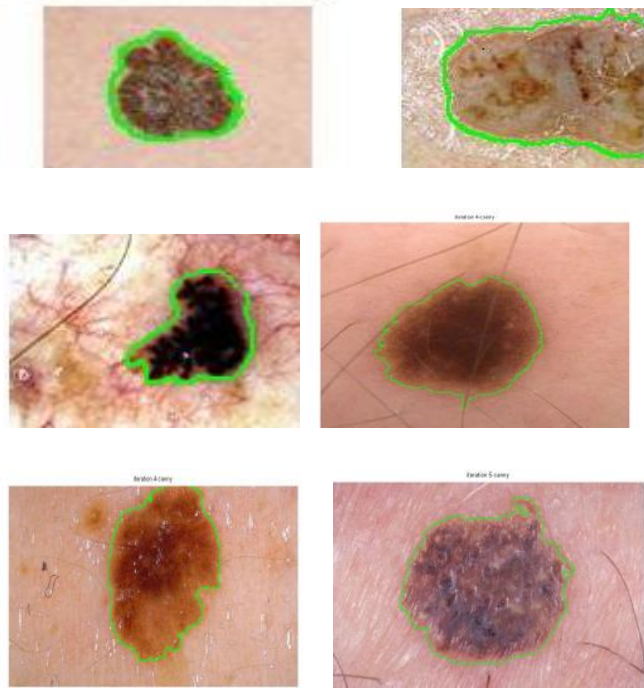

(b)

Fig 3b: Border detection results of various original skin lesions shown in Figure 2

(b) Final converged results obtained at different iterations for various original skin lesions by our proposed Improved Iterative Segmentation Algorithm using Canny Edge Detector with iterative filtering

Our aim is to select an image and the system should impart an automatic identification (or segmentation) of the lesion, which aims at identifying the lesion and separate it from the background.

Our proposed segmentation algorithm works well even in the presence of noise, to detect the border of the lesion. Fig 2 illustrates different types of original skin lesions. Fig 3a shows the final output results of the segmentation algorithm using canny edge detector [1], by J.H.Jaseema Yasmin et al. [1], when they are applied to the different types of skin lesions, with noise.

For the different types of skin lesions taken, J.H.Jaseema Yasmin et al. [1] method poorly delineates the boundary for of the skin lesions as shown in Fig 3a and demonstrates the failure of this method [1] to delineate the boundary of the lesion of various types

Our proposed improved iterative segmentation algorithm using canny edge detector, converts the original skin lesion image into a gray scale image. $10 \%$ salt and pepper noise was added to the original image. The noisy image is the input image to the proposed algorithm. The iterative median filter is applied and the noise is removed. After noise removal the image is enhanced. Based on a 
threshold value the enhanced image is converted to black and white image. This algorithm converts the black and white image into xor image and using canny edge detector, we get the edge detected image.

To improve the reliability of the algorithm in detecting the border of the skin lesions, the edge detected image is applied iteratively to the canny edge detector and for each iteration we get a border detected image. Then the various iterations at which we obtain the final converged results (skin lesion Border Traced image) for various original skin lesions by our proposed method are as shown in Fig $3 b$.

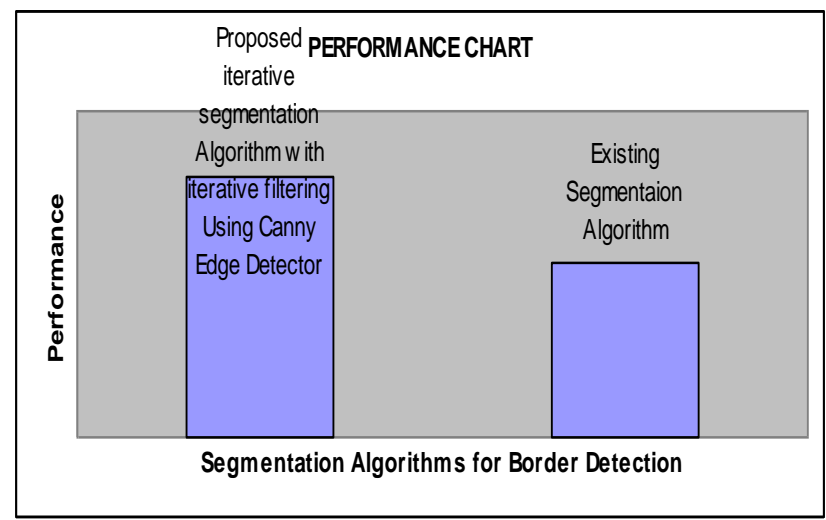

Fig 4: Performance comparison chart of the proposed Improved Iterative Segmentation Algorithm Using canny Edge Detector and existing Segmentation Algorithm Using canny [1] Edge Detector for tracing the border of noisy skin lesion images

The Performance comparison chart of the segmentation algorithm using the proposed algorithm and the segmentation algorithm using canny edge detector [1] for tracing the border of noisy skin lesion images is shown in Fig 4. The segmentation algorithm which uses canny detector [1] to trace the borders of the noisy skin lesion fails to detect the border for some of the original skin lesion images taken as shown in Fig 3a. The proposed segmentation algorithm successfully detects the border of the noisy skin lesions in all of the cases as shown in Fig 3b

So the performance of the proposed improved segmentation algorithm using canny edge detector with iterative filtering for tracing the border of noisy skin lesion images is better than the performance of the segmentation algorithm canny edge detector[1] for tracing the border of noisy skin lesion images.

\section{CONCLUSION}

In conclusion, this paper presents a simple yet effective border finding algorithm (improved iterative segmentation algorithm using canny edge detector with iterative filtering) for noisy skin lesions and it compares, its performance with that of the segmentation algorithm using canny detector [1] in the border detection of real noisy skin lesions. The detection of skin lesion boundaries accurately allows, skin cancer detection. There is no unified approach to this problem, which has been found to be application dependent. To validate the capability of the segmentation algorithm in detecting the border of the lesions for skin lesion diagnosis, the algorithm was applied on variety of clinical skin image containing lesions with noise. The experimental results demonstrated the successful border detection of real skin lesions by our proposed improved iterative segmentation algorithm using canny edge detector with iterative filtering for clinical skin lesion images with noise and make them available for further analysis and research. We conclude that our proposed improved iterative segmentation algorithm using canny edge detector with iterative filtering is successful in detecting the border of the skin lesions, even in the presence of noise for a variety of lesions, and skin types and we conclude that its performance is more reliable than the segmentation algorithm that uses canny detector [1], for border detection of noisy real skin lesions.

\section{REFERENCES}

[1] J.H.Jaseema Yasmin, M.Mohamed Sathik, S. Zulaikha Beevi, Effective Border Detection of Noisy Real Skin Lesions for Skin Lesion Diagnosis by Robust Segmentation Algorithm in IJARCS,Vol.1,No.3,Sept.Oct.,2010

[2] N. Senthilkumaran and R. Rajesh , Edge Detection Techniques for Image Segmentation - A Survey of Soft Computing Approaches in International Journal of Recent Trends in Engineering, Vol. 1, No. 2, May 2009

[3] S.Lakshmi, Dr.V.Sankaranarayanan, A study of Edge Detection Techniques for Segmentation Computing Approaches in IJCA Special Issue on "Computer Aided Soft Computing Techniques for Imaging and Biomedical Applications"CASCT, 2010.

[4] Jagadish H. Pujar, Pallavi S. Gurjal, Shambhavi D. S, Kiran S. Kunnur, Medical Image Segmentation based on Vigorous Smoothing and Edge Detection Ideology in International Journal of Electrical and Computer Engineering 5:2 2010

[5] C.NagaRaju ,S.NagaMani, G.Rakesh Prasad, S.Sunitha, Morphological Edge Detection Algorithm Based on Multi-Structure Elements of Different Directions in International Journal of Information and Communication Technology Research, Volume 1 No. 1, May 2011

[6] R. Harrabi, E. Ben Braiek ,Color Image Segmentation Based on a Modified Fuzzy C-means Technique and Statistical Features in International Journal Of Computational Engineering Research, Jan-Feb 2012 , Vol. 2, Issue No.1, Page 120

[7] Rahil Garnavi, Mohammad Aldeen, M. Emre Celebi, Alauddin Bhuiyan, Constantinos Dolianitis, and George Varigos, Automatic Segmentation of Dermoscopy Images Using Histogram Thresholding on Optimal Color Channels in International Journal of Biological and Life Sciences 8:2 2012

[8] M. Emre Celebix and Hassan A. Kingravi, Hitoshi Iyatomi, JeongKyu Lee, Y. Alp Aslandogan, William 
Van Stoecker, Randy Moss, Joseph M. Malters, Ashfaq A. Marghoob, Fast and Accurate Border Detection in Dermoscopy Images Using Statistical Region Merging

[9] Bilqis Amaliah, Chastine Fatichah, M.Rahmat Widyanto, ABCD Feature Extraction for Melanoma SkinCancer Diagnosis

[10] Meng-Husiun Tsai, Yung-Kuan Chan, Zhe-Zheng Lin, Shys-Fan Yang-Maob, Po-Chi Huang, Nucleus and cytoplast contour detector of cervical smear image in Pattern Recognition Letters 29 (2008) 1441-1453

[11] F. Ercal, M. Moganti, W. V. Stoecker, and R. H. Moss, Detection of Skin Tumor Boundaries in Color Images in IEEE transactions on medical imaging, Vol. 12, No. 3, September 1993

[12] L. Xu, M. Jackowski, A. Goshtasby, D. Roseman, S. Bines, C. Yu, A. Dhawan, A. Huntley, Segmentation of skin cancer images in Image and Vision Computing 17 (1999) 65-74

[13] S.Zulaikha Beevi, M.Mohamed Sathik, A Robust Segmentation Approach for Noisy Medical Images Using Fuzzy Clustering With Spatial Probability in European Journal of Scientific Research ,Vol.41, No.3 (2010), pp.437-451
[14] S.Zulaikha Beevi*,M.Mohammed Sathik, K.Senthamarai Kannan, J.H Jaseema Yasmin, Hybrid Segmentation Approach using FCM and Dominant Intensity Grouping with Region Growing on Medical Image in International Journal of Advanced Research in Computer Science, Volume 1, No. 2, July-August 2010

[15] Maciel Zortea, Stein Olav Skrøvseth, Thomas R. Schopf, Herbert M. Kirchesch, and Fred Godtliebsen, Automatic segmentation of dermoscopic images by iterative classification, International Journal Of Biomedical Imaging ,pp.1-17

[16] Nhi H. Nguyen, Tim K. Lee, M. Stella Atkins, Segmentation of light and dark hair in dermoscopic images: a hybrid approach using a universal kernel

[17] German Capdehourat, Andres Corez, Anabella Bazzano, and Pablo Muse, Pigmented skin lesions classification using dermatoscopic images

[18] M.Emre Celebi, Hitoshi Iyatomi , Gerald Schaefer, William V. Stoecker, Lesion border detection in dermoscopy images, Computerized Medical Imaging and Graphics $33 \quad$ (2009) 148-153 